\title{
LAS ESPERANZAS DE PANDORA: PROTOTIPOS FEMENINOS EN LA OBRA DE ROSARIO FERRÉ
}

POR

\author{
LORRAINE ELENA ROSES \\ Wellesley College
}

o por esas hembras palúdicas que depositan el silencio en una caja de hierro a cambio de comida. Lourdes Vázquez, "Las hembras”.

Una lectura diacrónica de la obra de Rosario Ferré, nos muestra una preocupación constante y creciente por Puerto Rico y por los drásticos cambios de orden socio-político y económico que ha vivido la isla desde 1898. Esto es evidente en ensayos tales como "La cocina de la escritura" y "On Love and Politics" en los que Ferré habla de un mundo en crisis y donde define la "dependencia y la fragmentación" prototípicas de la condición puertorriqueña. El enfoque casi obsesivo en esta problemática no es exclusivo de Ferré, sino que se ve compartido en mayor o menor grado por la mayoría de los intelectuales y artistas de su generación. ${ }^{1}$ Pero Ferré extiende su interpretación de la realidad puertorriqueña, exigiendo que esté presente la mujer en el colectivo diálogo del proceso histórico puertorriqueño.

En Sitio a Eros (1980) Ferré examina el problema de la mujer como escritora, sobre todo la actitud de la mujer europea, partiendo de las ideas de Simone de Beauvoir y Virginia Woolf. El análisis que la autora hace sobre la escritura de las grandes mujeres (Mary Shelley, Lillian Hellman, Virginia Woolf, Jean Rhys, Julia de Burgos, y otras) está centrado en una dialéctica de la mujer creadora ante la sociedad patriarcal que le frustra su afán de realización y de creación. Al dar una visión de la escritora que sufre ante su vocación, Ferré une la idea del poder femenino a la del amor, insistiendo que la mujer es un ser intuitivoy pasional. Aunque este libronoincluye la problemática de la creación de la mujer del tercer mundo, Ferré lo escribe por y para ella, con la intención de que aprenda de sus predecesoras. Por el contrario, en su obra de ficción despliega conscientemente una visión del mundo puertorriqueño desde la perspectiva de la mujer.

\footnotetext{
${ }^{1}$ Para una definición del concepto generacional referido a Puerto Rico, ver de Efraín Barradas, el "Prefacio" de Apalabramiento: cuentos puertorriqueños de hoy.
} 
En Papeles de Pandora (1976) aparecen unidos los dos temas que obsesionan a la autora - política y mujer-queriendo con ello dilucidar para sí misma la resolución de un conflicto. En este libro nos da una visión de un sistema cultural dominado por los valores patriarcales que a su vez sufren la colonización de unos valores foráneos y alienantes. Ciertamente, esta actitud inquisitiva de los conflictos patriarcales y del coloniaje no siempre le han traído a Ferré el elogio del público; por el contrario, como ella verifica en una entrevista de 1983 (García Pinto), el primer número de la revista Zona de carga y descarga, co-fundada por ella, fue quemada en los patios de algunas casas, y la presencia de Ferré en los periódicos, por mínima que sea, suele causar el horror familiar. Esta reacción pública no es más que un mero exponente actual de las dificultades que históricamente ha sufrido toda mujer que se quiera hacer autora/autoridad. Como la misma Ferré puntualiza en sus ensayos, "La mujer con vocación literaria no llegará hoy acaso a [los extremos históricos citados por Virginia Woolf] pero sigue estando muy lejos de tener una suerte tranquila: su vida se vuelve una vorágine de conflictos que intentan destrozarla en la medida en que persiste en realizar la voz de su corazón, o sea, su vocación" (Sitio a Eros, 13).

Estas adversidades se ven recapituladas en Hispanoamérica con Alfonsina Storni, Juana de Ibarborou y Julia de Burgos, sin evocar el caso ejemplar de Sor Juana Inés de la Cruz. El caso de Ferré es sin embargo singular, porque ella pertenece a una familia distinguida e influyente en la ciudad de Ponce que ha desempeñado un papel decisivo en los destinos puertorriqueños.

Por lo tanto, la palabra de Rosario Ferré, a pesar de la marginación que sufre por razón de su sexo, emana desde el centro mismo del sistema expresivo del poder. ${ }^{2}$ Esto lleva a la autora a una ambivalencia que ella misma reconoce cuando lamenta la pérdida de una sociedad cañera que estaba basada en la explotación pero que incluía también "una serie de valores" y una "identidad" propias. Ferré se fija sobre todo en

la pérdida de ciertos valores ... [como] el abandono de la tierra; el olvido de un código de comportamiento patriarcal, basado en la explotación, pero también a veces en ciertos principios de etica y de caridad cristiana sustituidos por un nuevo código mercantil y utilitario que nos llegó del norte ... ( ${ }^{4 L} \mathrm{La}$ cocina de la escritura", 41).

Desde la postura céntrica que ella ocupa por su clase social, el criticar los absurdos y los abusos del establishment le crea a Ferré un problema de lealtad y de identidad. Por lo tanto es necesario afirmar que el mantenimiento de esta postura heterodoxa, por ambivalente que sea, requiere un gran atrevimiento. La autora resuelve (disuelve) su ambivalencia trascendiendo su crítica social

\footnotetext{
${ }^{2}$ Hija de Luis A. Ferré, fundador del Partido Nuevo Progresista y gobernador de Puerto
} Rico de 1968 a 1972, Rosario adhiere a una postura política divergente: la independendista. 
hacia lo existencial. Dicho de otra manera, su crítica asume una dimensión radical de autocrítica personal y de "invertebración" nacional.

Estos conflictos entre el poder patriarcal, del que participa por su posición social, y su marginación como mujer desautorizada son expresados por Rosario Ferré a través de múltiples prototipos femeninos, entre los cuales se incluyen la muñeca, el doble, la inválida, la hechicera y la histérica. Lo que me interesa estudiar con respecto a Papeles de Pandora, a la luz de algunos estudios de teoría feminista, son estas dos últimas, la hechicera y la histérica. Con el escrutinio de ambas quisiera proponer una nueva lectura de dos cuentos muy comentados de Rosario Ferré. Hasta ahora la crítica, por lo demás bastante nutrida, de la cuentística de Ferré, se ha concentrado en cuestiones estilísticas y formales, tales como el doble (Lagos Pope) o la muñeca (Sánchez, López Jiménez). Mi intención es sugerir una lectura cultural, apoyada en las dos figuras mencionadas anteriormente, y que sea complementaria a otros posibles acercamientos.

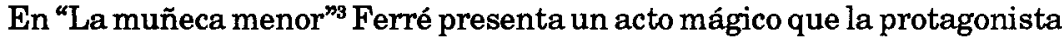
realiza para sustraerse a una larga subyugación. Esta mujer ha sido infectada por un parásito ("chágara") marino, y un médico explota su herida diciéndole que no tiene cura. Ante esta sentencia ella se resigna a la soltería y, a partir de entonces, a criar a sus nueve sobrinas. Para cada sobrina, ella cose una muñeca cuyos ojos son joyas, y el proceso va llenando la casa hasta que, una a una, las sobrinas se las llevan, como dote, a su nuevo domicilio. La más pequeña de las sobrinas acepta casarse sin amor con el hijo del médico, quien aspira a aliarse con esa familia aristocrática. Siguiendo el ejemplo del padre, que había despojado a la tía de su dinero, el joven médico quiere dilapidar también la dote de su esposa. Para ello arranca las joyas de los ojos de la muñeca con la intención de venderlos. En esto hay una fusión entre muñeca y mujer. No se sabe si la hechicería viene de la tía o de la sobrina, cosa que sugiere al lector la idea borgiana de que tal vez todas las mujeres sean la misma mujer.

Por otra parte, en otro cuento, "Cuando las mujeres quieren a los hombres", se ve un caso de histeria, si por histeria entendemos el padecimiento psíquico historiado por Freud y sus seguidores. ${ }^{4}$ Dos mujeres en los extremos de la escala social (siendo una prostituta/madama y la otra dama de sociedad) chocan en un encuentro sangriento por el hecho de ser éstala esposa de un hombre distinguido, y aquélla la amante. La mujer legítima, que ha sufrido por años de una rivalidad obsesiva con la otra, se enfrenta con ella y estalla violentamente toda su furia, mientras que las dos voces su funden en una sola. El comportamiento que Ferré describe podría relacionarse desde un punto de vista psicoanalítico como una reacción histérica, y por extensión, con el discurso caótico (histérico) que caracteriza el relato.

\footnotetext{
${ }^{3}$ Todas las citas de cuentos de Ferré proceden de Papeles de Pandora. Manejo la primera edición, de 1976.

${ }^{4}$ Véase por ejemplo Freud, Dora: An Analysis of a Case of Hysteria.
} 
El planteamiento que hace Ferré de ambas figuras, la hechicera y la histérica, connota una crítica directa hacia el orden patriarcal y las costumbres que la sostienen, entre ellas la ilimitada libertad sexual reservada para el hombre y el mantenimiento de la mujer en la dependencia sexual y económica. Al mismo tiempo, estos dos personajes pueden concebirse como ejes de toda la colección Papeles de Pandora.

Es interesante notar que estas figuras, la de la hechicera y la histérica, han merecido el acucioso estudio también de otras escritoras. Por ejemplo la francesa Catherine Clement, co-autora con Helene Cixous de La jeune née (1976), asevera que estas dos figuras surgen repetidamente en la literatura y en el psicoanálisis como una representación de lo reprimido. Siguiendo a Marcel Mauss y Levi-Strauss, dice Clement que lo reprimido es relegado a una "zona imaginaria" que existe en el inconsciente cultural para lo que la sociedad quiere reprimir y excluir. Fijándose en textos escogidos de Flaubert, Michelet, y Freud, Clément reinterpreta la hechicería y la histeria como símbolos de un pasado reprimido, cuya expresión es canalizada a través de la mujer. A causa de que porta en ella valores sancionados por la sociedad, la mujer es de un extraordinario potencial subversivo. La hechicera (o la vulgarmente llamada bruja) sabe curar, hacer abortar, propiciar el amor ilícito, y aún conminar el dominio de la religión (Clément, 5), por lo que se convierte en un elemento peligroso para el orden patriarcal y termina no siendo registrada por la historia. Asimismo, por vivir apartada de los hombres y sin familia fue siempre perseguida de igual manera que ahora empieza a ser considerada como precursora de la resistencia feminista.

La histérica, a su vez, encarna la reminiscencia obstinada del pasado, borra las estructuras establecidas, e introduce y fomenta el desorden. Ambas representan lo anti-racional, lo antijerárquico, y los impulsos reprimidos, pero no borrados por la cultura patriarcal. Dice Clément:

The heart of the story linking the figures of sorceress and hysteric lies in the subversive weight attributed to the return of the repressed, in the evaluation of the power of the archaic and in the Imaginary's power or lack of it over the Symbolic and the Real" (9).

Así, lo intuitivo y lo anti-racional pueden identificarse con lo femenino. Yendo más allá, en esta segregación de la mujer está la idea de que la mujer se identifica y vive unida a la naturaleza y, por lo tanto, en el inconsciente colectivo resulta inferior al hombre, cuyo dominio es la civilización. Las sociedades valoran más lo masculino que lo femenino por la preeminencia de lo cultural. De ahí que se valore también más el desempeño público donde imperan los hombres, que el privado, regido por las mujeres. ${ }^{5}$

${ }^{5}$ La situación física, social y psicológica de la mujer hace que se la perciba como cercana a la naturaleza. Esta percepcion a su vez se encarna en forma institucional que reproduce la situación de inicio. Así lo analiza Sherry B. Ortner en su ensayo "Is Female to Male as Nature is to Culture?" 
Ya desde el título es obvio que Papeles de Pandora se centra en la revisión de lo que es lo femenino, siendo Pandora el nombre de un avatar greco-latino de Eva, símbolo de la feminidad esencial. Mujer rebelde que por orden de Zeus suelta en el mundo la enfermedad, la prematura vejez, el trabajo duro y otros males, también es Pan/Dora, la toda generosa de la fábula original, que tuvo que ceder a un panteón masculino. ${ }^{6}$ Por otra parte, como ya ha señalado Luz María Umpierre, el título polisémico sugiere los papeles personales de una narradora mujer; a la vez indica los distintos "papeles oroles quelas mujeres se ven obligadas a desempeñar ... en la vida" que Ferré quiere denunciar en sus cuentos (Umpierre, 121). Pandora, por orden y maldición de Zeus, libera una carga de furia contra el mundo, así como Ferré también se apodera de la escritura, transgrediendo el tabú ancestral que le prohibía el acceso a la mujer.

Volviendo a la obra de Ferré, vemos que Papeles de Pandora, apartándose de los patrones europeos, hace eco de un proceso doble de patriarcalismo y de coloniaje. En este mundo caribeño, negación, como el Macondo próximo a la caída, de míticos paraísos, la forma del poder establecido se presenta como algo abusivo. En este contexto el erotismo desplaza el amor y la atmósfera sofoca en vez de dar vida; igualmente la relación amorosa entre un hombre y una mujer se convierte en una dinámica de dependencia y explotación mutua. En tal ambiente brillan por su ausencia las pintorescas palmeras que otrora sirvieran como telón de fondo para idilios de brisa y mar, como en el caduco locus amoenus de la imaginación europea. ${ }^{7}$ Se borran tanto el pasado como el futuro, y hay una entrega total a la apatía. En otros cuentos incluidos en Papeles de Pandora Ferré describe paisajes cálidos y polvorientos, jardines descuidados, laberintos y objetos inútiles que aluden a una destrucción apocalíptica. Nos encontramos dentro de una atmosfera de "triste tropicalismo", en un orbe poblado de personajes pasivos, autoindulgentes y blandos ante una invasión que reitera conquistas anteriores. Al verse inmovilizados por su propia abulia, estos seres endebles, que encarnan la “docilidad” definida por Antonio Pedreira y René Marqués comoidiosincracia isleña, ni crean ni sueñan, sino que se descomponen como frutas tropicales. Los personajes y el escenario se interpenetran y se reflejan infinitamente como en espejos enfrentados. No es casual que en este mundo se inserten la hechicería y la histeria.

Dentro del contexto caribeño, la hechicería resuena de una forma distinta que en el mundo europeo. Evoca formas y ritmos sincretizados con energías telúricas. En "La muñeca menor" el acto de confeccionar muñecas entraña materiales recogidos de la naturaleza tropical:

Para hacer el cuerpo, la tía enviaba al jardín por veinte higueras relucientes. Las cogia con una mano y con un movimiento experto de la cuchilla las iba

\footnotetext{
6 Según la interpretación de Robert Graves.

${ }^{7}$ Esta dicotomía referente al Caribe aparece en un ensayo de Edmundo Desnoes, "El Caribe: paraiso/infierno".
} 
rebanando una a una en cráneos relucientes de cuero verde. Luego las inclinaba en una hilera contra la pared del balcón para que el sol y el aire secaran los cerebros algodonosos de guano gris. Al cabo de algunos días raspaba el contenido con una cuchara y lo iba introduciendo con infinita paciencia por la boca de la muñeca (11-12).

Hay abundantes evidencias de que mujer y naturaleza se unen en el acto de la confección. Es más, en la muñeca se conjugan las fuerzas vitales y mortíferas, pues la tía "... hacía una mascarilla de cera que cubría de yeso por ambos lados como una cara viva dentro de dos caras muertas" (12). Así vemos que su sentido no es otro que un poder oculto, proveniente de las zonas más mágicas del ser. En el desenlace horrorífico que emparenta el cuento con la literatura fantástica ${ }^{8}$ estos poderes son activados por el médico desalmado al inclinarse para examinar a su mujer:

Colocó delicadamente el estetoscopio sobre su corazón y oyó un lejano rumor de agua. Entonces la muñeca levantó los párpados y por las cuencas vacías de los ojos comenzaron a salir las antenas furibundas de las chágaras.

Así se revela la muñeca comouninstrumentolabrado para una reivindicación espiritual a través de los únicos medios accesibles a la mujer. Se libera el potencial para la brujería, expresión metafórica de una cólera antigua y apenas contenida que al final acaba dirigiéndose contra el conquistador.

Así como la hechicería rompe con el orden jerárquico y logocéntrico para instaurar otra hegemonía procedente de la naturaleza, la histeria derriba todas las pretensiones y expone la hipocresía de la exclusión y el marginamiento. Si la histérica es la que llora, se descontrola, se desborda, en su padecimiento se trasparenta la realidad de la opresión de la mujer.

Visto de esta manera, el cuento "Cuandolas mujeres quieren a los hombres" es una metáfora para el silenciamiento de la mujer, a quien no se le permite protestar contra el desamor y la infidelidad. La crítica se ha concentrado más bien en la presencia del doble y otras proyecciones técnicas y estilísticas en el relato. Ciertamente, el desdoblamiento de la dama de sociedad en prostituta, o viceversa, configura hasta cierto punto el relato. Sin embargo, el punto culminante del mismo sólo se alcanza a través de un mecanismo de ruptura, o sea el momento histérico en que la elegante y ejemplar señora acomete a la prostituta en un ataque sangriento. No le han valido sus intentos de recuperar el amor de su marido a través de las tretas de la brujería, como aplicar gotas de limón a su servilleta, perfumar las sábanas o "prender cuatro velas por los rincones para ver quien ganaba".

\footnotetext{
${ }^{8}$ Véase el excelente análisis que ofrece Yvette Lopez Jiménez del discurso fantástico en "La muñeca menor".
} 
Desde el comienzo, las palabras "desbarajuste", "escándalo", y "confusión" apuntan a la ruptura con las normas fundamentales de creencia y de conducta, de la misma manera que la presencia del llanto agrieta la apariencia de la felicidad. Isabel, la esposa, se pone a llorar al contacto físico con la querida de su marido, como lloró durante muchos años de matrimonio: "De tanto llorar parecía que me hubiesen inyectado coramina en el interior de los párpados". El momento del llanto, que también marca la fusión mágica de dos mujeres diametralmente opuestas en la escala social, borrándose las fronteras de la personalidad, remite nuevamente a la hechicería vista en "La muñeca menor".

Si la esposa recurre a la magia, la amante también es "sacerdotisa" del sexo, que incita a la locura y al erotismo, susurrando a los hombres que "... no hay nada prohibido, el cuerpo es el único Edén sobre la tierra, la única fuente de las delicias". Así se llegan a asociar en la mente del lector los dos prototipos que estamos examinando y se explica el enorme poder de absorción que acaba fundiendo a las dos mujeres en un acto de "purificación" casi religiosa.

La fusión es llevada a cabo también por el deseo que siente la una de la otra, introduciendo así la idea de la bisexualidad. Una bisexualidad que tiende a corroer la oposición tajante entre lo femenino y lo masculino y subvertir el machismo. Cuando Isabel Luberza, desilusionada de los hombres, siente "deseos de besarle los párpados [a su rival], tiernos como tela de coco nuevo y rasgados a bisel" y hasta "lamérselos" (34) nos aproximamos al terreno de una androginia cuya función es cuestionar la rigidez de las categorías sexuales.

Estos dos cuentos de Papeles de Pandora pueden considerarse como los ejes de un enunciado que imprime y refuerza la idea del título de la colección. Las figuras de la hechicera y de la histérica, por su significado cultural dentro de la tradición histórico-literaria, cobran una nueva dimensión al ser situadas por Ferré en un contexto caribeño y concretamente puertorriqueño. A diferencia de la interpretación del pasado y el sueño de la utopía que nos ofrecen Hélene Cixous y Catherine Clément, Rosario Ferré nos entrega una visión de una existencia cautiva en las redes del coloniaje y el patriarcado. Hechicería, como forma alterna y subterránea de cosmovisión e histeria, como desviación y rechazo del patriarcado, adquieren matices radicales en el contexto caribeño, es decir, una dimensión feminista y anti-colonial que destaca la originalidad de la escritura de Ferré.

Sería difícil determinar si Rosario Ferré se suscribe a un futuro en que la mujer, por medio de su palabra y de su escritura, saldrá de su marginamiento; en el que Puerto Rico sacudirá las cadenas del coloniaje. La narrativa de Ferré oscila entre la afirmación atrevida de los poderes del marginado (vertiente positiva de la hechicería) y un concepto que insinúa que el destino isleño es, hasta cierto punto, producto de un letargo y de una frustración permanentes (vertiente negativa). Se presenta la paradoja de la mujer como víctima y enemiga de sí misma, tal como la veía en "Los soles truncos" René Marqués. Ferré no nos deja leer a la mujer protagonista de su propia vida - sea como 
matriarca, bruja, reformista u obrera. Lo que sí permite descifrar es un subtexto de rabia y de amargura postergadas. ¿Se trata de una visión que tiende hacia el pasado o que de alguna manera apunta hacia el futuro? Otra vez topamos con la ambivalencia entre la vuelta a un pasado con valores estables y la instancia hacia un futuro desconocido y ajeno. La confluencia de ambas tendencias ocurre en la mente del lector, incitando a una dialéctica permanente.

Estas ambigüedades noquitan que Ferré se haya enfrentado resueltamente a la problemática de la mujer y de su país. En su obra los avatares de la hechicera y la histérica funcionan como metáforas globales para los usos del poder y la apertura al cambio. Sin rehuir los temas de la crueldad, la vulgaridad y la violencia, Ferré abre nuevos caminos para la interpretación de la realidad que le ha tocado vivir y nos invita a nosotros lectores a acompañarla en su viaje hacia lo incógnito.9

\section{Obras citadas}

Barradas, Efraín. ed. Apalabramiento: Cuentos Puertorriqueños de hoy. Hanover, New Hampshire: Ediciones del Norte, 1983.

Cixous, Hélene, and Catherine Clément. The Newly Born Woman [1975]. Trad. Betsy Wing. Minneapolis: University of Minnesota, 1986.

Desnoes, Edmundo. "El Caribe: paraíso/infierno". Minc, Rose, ed. Literature in Transition: The Many Voices of the Caribbean, A Symposium, 9-16.

Ferré, Rosario. "On Love and Politics”. Review: LatinAmerican Literature and Arts 37 (enero-junio 1987), 8-10.

Ferré, Rosario. Papeles de Pandora. México: Joaquín Mortiz, 1976.

Freud, Sigmund. Dora: An Analysis of a Case of Hysteria. Nueva York: MacMillan, 1963.

García Pinto, Magdalena. Historias íntimas: conversaciones con diez escritoras latinaoamericanas. Hanover, New Hampshire: Ediciones del Norte, 1988. Gascón Vera, Elena. "Rosa Montero ante la escritura femenina". Anales de la Literatura Española Contemporánea 12 (1987) 59-77.

Graves, Robert . The Greek Myths. Baltimore: Penguin Books, 1955.

Lagos Pope, María Inés. "Sumisión y rebeldía: el doble o la representación de la alienación femenina en narraciones de Marta Brunet y Rosario Ferré". Revista Iberoamericana 132-133 (jul-dic 1985) 731-749.

López [Jiménez], Yvette. "La muñeca menor": ceremonias y transformaciones en un cuento de Rosario Ferré. Explicación de Textos literarios 1 (1982-83) 49-58.

\footnotetext{
- Agradezco a mi estimada colega Elena Gascón Vera, cuyos ensayos sobre la escritura femenina me inspiraron para elaborar este trabajo y cuyos comentarios me han sido muy valiosos.
} 
Ortner, Sherry B. "Is Female to Male as Nature is to Culture?" in Michelle Zimbalist Rosaldo and Louise Lamphere, eds., Women, Culture, and Society (Stanford, California: Stanford University Press, 1974) 67-88.

Sánchez, Luis Rafael. [reseña de Papeles de Pandora]. Claridad 21-27 (Abril, 1978) 21.

Umpierre, Luz María. "Un manifiesto literario: Papeles de Pandora de Rosario Ferré", Bilingual Review 2 (May-August, 1982) 120-126. "La nueva mujer ... surgirá de la destrucción de su antiguo ser social"

Vázquez, Lourdes. Las hembras . N.Y.: Papeles del Andalicán, 1986. 
